\title{
Editorial: Embodied learning in an online world
}

\author{
Alicja Syska \\ University of Plymouth
}

One of the greatest challenges the pandemic brought to the forefronts of the educators' minds across the globe was the reality of experiential and embodied learning, which often requires kinetic and tactile involvement with objects, places and spaces, alongside discursive in-class engagements. Merleau-Ponty's reminder that 'rather than a mind and a body man [sic] is a mind with a body, a being who can only get to the truth of things because its body is, as it were, embedded in those things' (2004, p. 43) has never rung more true than when we were denied this embodied experience and became separated by (often blank) screens, increasingly relying on technology. Ironically, it was technology that we had little choice but use to either mimic or reinvent this embodied experience.

In this section of the Compendium, our writers-educators report on how they had to deal with the most literal aspects of what we understand as embodied learning experience. To them, it was not just about broadening our thinking about teaching by acknowledging that our bodies matter and that privileging cognitive and abstract learning may limit students' educational experiences. Indeed, the challenges for these authors involved recreating activities such as handling physical objects to teach about history and material culture; reinventing play, collage making, and drawing in teacher training; reprioritising the body when teaching positive psychology and while delivering lectures to the screen; rethinking hands-on instruction of anatomical structures and performing cadaveric dissections in medicine; as well as redesigning hospital placements, mock crime scenes and intercultural field trips.

What you will see in these reports is a fabulous range of approaches and an inspiring creativity with which these authors responded to the very particular challenges they faced. What they demonstrate is that objects can be replaced with well-designed handouts; real archives with virtual trips to these archives; discursive interactions with interactive digital 
resources; outward-reaching strategies with inward body-based approaches; lecturing 'to the screen' with lecturing 'through the screen' (Morris, 2020); physical dissections with 3D models and videos; clay and paper anatomy with Minecraft modelling; mock crime scenes with virtual crime scenes; and intercultural field trips with virtual field trips. For some educators, it was possible to retain the bodily/experiential element through home tasks at the expense of the shared experience involved in creating synchronously, while others used social distancing regulations to improve their processes and provide access to physical learning spaces such as museums for a limited number of learners.

Together, what these pieces show is that playful experimentation, openness to creative ways of teaching, and dialogic approaches can result in a student experience that is not detached but embodied, and which encourages exploration and agility. Even if most authors conclude that there are no viable alternatives to certain embodied learning activities, they all admit that they made unusual and productive discoveries about the disruptive potential of the online pivot to challenge both their students' and their own normative expectations and conventional thinking around education.

\section{References:}

Merleau-Ponty, M. (2004 [1948]) The world of perception. London and New York: Routledge.

Morris, S.M. (2020) 'Teaching through the Screen and the Necessity of Imagination Literacy', Sean Michael Morris Blog. 3 December. Available at https://www.seanmichaelmorris.com/teaching-through-the-screen-and-the-necessityof-imagination-literacy/ (Accessed 10 October 2021). 